DOI: https://doi.org/10.46296/yc.v4i6edesp.0026

\title{
MODELO DE GESTIÓN HOTELERA DE LA CIUDAD DE PORTOVIEJO
} PORTOVIEJO CITY HOTEL MANAGEMENT MODEL

\author{
Serrano-Amaya Sara Elizabeth ${ }^{1 *}$; Poveda-Cedeño Eliana ${ }^{2}$ \\ ${ }^{1}$ Estudiante de la Maestría de Administración de Empresas/Instituto de \\ Posgrado/Universidad Técnica de Manabí. Portoviejo, Ecuador. \\ ${ }^{2}$ Magíster en Administración de Empresas. Profesora de la Facultad de Ciencias \\ Administrativas y Económicas, Universidad Técnica de Manabí. Portoviejo, Ecuador.
}

*Correo: saraserrano6609@gmail.com

\begin{abstract}
Resumen
La presente investigación se fundamenta en el análisis del sector hotelero de Portoviejo, Manabí, Ecuador; puesto que es bien conocido que esta ciudad posee atractivos turísticos asociados con la gastronomía, cultura y recursos naturales. Por lo tanto, el objetivo de la presente investigación fue diseñar un modelo de gestión hotelera en la ciudad de Portoviejo para garantizar la sostenibilidad turística. En este sentido, se utilizaron estrategias metodológicas de tipo cuantitativo y cualitativo, así como la revisión bibliográfica de manuscritos y reportes oficiales para dar cumplimiento a los objetivos planteados. En total, la muestra estuvo compuesta por 67 representantes o administrativos de establecimientos hoteleros, que representa el $82,7 \%$ del número de hoteles de la ciudad. Se determinó que el $41,5 \%$ de los turistas manifiestan que llegan por descanso y vacaciones. Por otra parte, es de carácter imperioso que los gobiernos autónomos descentralizados cantonales como provinciales aporten herramientas en materia de formación y capacitación hacia la alta directiva y los trabajadores de los hoteles, puesto que, el $63,89 \%$ de los encuestados indicó que no han sido capacitados. Se concluye que, si bien la gestión hotelera ha permitido potenciar la actividad turística de Portoviejo, aún desde las instituciones públicas no se entregan herramientas y mecanismos suficientes para que los establecimientos hoteleros brinden un servicio de calidad y alcancen la sostenibilidad de sus actividades comerciales.
\end{abstract}

Palabras clave: gestión, hotelería, planificación, turismo, sostenibilidad.

\begin{abstract}
This research is based on the analysis of the hotel sector of Portoviejo, Manabí, Ecuador; since it is well known that this city has tourist attractions associated with gastronomy, culture and natural resources. Therefore, the objective of this research was to design a hotel management model in the city of Portoviejo to ensure tourism sustainability. In this sense, methodological strategies of quantitative and qualitative type were used, as well as the literature review of manuscripts and official reports to comply with the objectives set. In total, the sample consisted of 67 representatives or administrators of hotel establishments, which represents $82.7 \%$ of the number of hotels in the city. It was determined that $41.5 \%$ of participants say they arrive for rest and vacations. On the other hand, it is imperative that autonomous cantonal decentralized and provincial governments provide tools for training and training towards senior management and hotel employees, since $63.89 \%$ of respondents indicated that they have not been trained. It is concluded that, although the hotel management has allowed to enhance the tourist activity of Portoviejo, even from the public institutions there are not enough tools and mechanisms provided so that the hotel establishments provide a quality service and achieve the sustainability of their commercial activities.
\end{abstract}

Información del manuscrito:

Fecha de recepción: 26 de febrero de 2020

Fecha de aceptación: 20 de abril de 2020

Fecha de publicación: 05 de mayo de 2020 
Keywords: management, hospitality, planning, tourism, sustainability.

\section{Introducción}

La complejidad del sector turístico se debe a que abarca tanto las actividades o los subsectores que resultan ser sistemas heterogéneos $y$, por otro lado, existen complejas y múltiples interrelaciones entre los elementos que lo constituyen (Huéscar, 1993). Según la Organización de las Naciones Unidas (ONU), en su comisión de estadística propone la siguiente definición: El turismo abarca "las actividades que realizan las personas durante sus viajes $y$ estancias en lugares distintos al de su entorno habitual, por un período de tiempo consecutivo inferior a un año, con fines de ocio, por negocios y otros motivos" (ONU, 1994).

La gestión hotelera comprende una serie de procesos que involucra el funcionamiento y operatividad de un hotel, estos procesos buscan la optimización y mejora continua de los procedimientos operativos del centro de alojamiento. Históricamente, se ha considerado que la gestión hotelera abarca tan solo objetivos asociados con el servicio a los clientes, no obstante, en la actualidad toma en cuenta aspectos socio-económicos del mercado, preferencias que guardan relación entre la bebida y comida. En este sentido, factores como la calidad del alojamiento y la restauración influyen individualmente en la rentabilidad de la empresa y por consiguiente, deben ser parte del desarrollo e implantación de un modelo de gestión. Castelló (1993) menciona las siguientes características elementales del servicio hotelero, se debe considerar que el producto que se oferta se fundamenta en la prestación de un servicio no inventariable una vez concluido, a pesar de que pueda serlo en un punto anterior del proceso. Evidentemente, la mayoría de los servicios tienen una caducidad inmediata 0 instantánea. Esta problemática o incluso imposibilidad de almacenar el producto o servicio insta a la industria hotelera a preocuparse por distribuir toda la producción diariamente, debido a que lo que no se vende puede definirse como una pérdida. El servicio no debe ser trasladado al cliente, por lo tanto, la empresa debe centralizar su atención en función del cliente y adaptar el servicio a las 
necesidades y circunstancias del entorno. El sector hotelero tiene la restricción de un incremento en la oferta de alojamiento, por ende, en caso de que aumente la demanda, el hotel no puede satisfacerla en el corto plazo (Musa \& Sanchís, 1993).

Además, la industria hotelera debe ofrecer una diversidad de servicios; las actividades relacionadas con la industria hotelera deben ser heterogéneas en función de los productos o servicios ofertados. Por otra parte, el trato o la actitud que el talento humano del hotel mantenga con los usuarios o consumidores es fundamental y decisivo (Ruhanen, 2008; Waligo et al., 2013).

Respecto a la economía y administración hotelera, se resalta que los costos fijos son muy altos en contraste con los costos variables. Además, la permanencia de la operatividad en durante todo el día (24 horas) altera el volumen del personal que labora, por tanto, es necesaria una planificación de las actividades con la finalidad de cubrir todas las necesidades. Mientras tanto, la diversidad geográfica de los clientes, así como la diversidad de mecanismos empleados para la contratación de servicios, son características que definen la industria hotelera (Aguayo, 1997).

Ecuador es un país que busca posicionarse como un destino turístico con una visión que permita potenciar la economía y aumentar los ingresos, de modo que cuenta con potencial natural, cultural y gastronómico. El turismo se ha posicionado como el tercer rubro en el Producto Interno Bruto (PIB), con una generación de 374,2 millones de dólares (Ministerio de Turismo, 2018).

Los aspectos mencionados previamente resaltan la importancia y dificultad que conlleva la administración hotelera y una adecuada gestión. Si bien muchos hoteles contratan a un asesor externo para realizar la gestión hotelera de su centro de alojamiento, aún en nuestro medio no existe una cultura de gestión hotelera que permita optimizar los recursos y adecuadamente cubrir las necesidades del cliente y el entorno. Por lo tanto, el objetivo general del presente trabajo es diseñar un modelo de gestión en la ciudad de Portoviejo para garantizar la sostenibilidad turística. Mientras que los objetivos específicos planteados 
son: i) caracterizar la gestión hotelera de los establecimientos ubicados en Portoviejo, ii) diagnosticar los principales inconvenientes en el manejo administrativo, operativo y de servicios en los hoteles de la ciudad de Portoviejo; iii) diseñar opciones de manejo administrativo, operativo y de servicios para los hoteles de la ciudad de Portoviejo.

\section{Materiales y métodos}

\subsection{Evaluación de la gestión hotelera}

Agatón y Cruz (2015) mencionan que es necesario realizar una definición de la zona de estudio, por su parte, Albuja (2016) proponen un modelo que considera el diagnóstico y la evaluación de potencialidades de los lugares turísticos; además, se reportan aspectos como la presentación de los resultados obtenidos (Batista et al., 2017). En este trabajo de investigación se tomaron cada una de las etapas propuestas por los autores mencionados.

\subsubsection{Fase de diagnóstico turístico}

Se consideraron parámetros como la delimitación de la zona, mediante mapeo y coordenadas del lugar de estudio; en cuanto al análisis de oferta, se realizó una evaluación de los factores que pertenecen al sistema turístico de Portoviejo, es decir los componentes turísticos que potencian la actividad hotelera. Cabe indicar que la calidad y gestión de los productos y servicios ofertados por el sector hotelero fue evaluado mediante una encuesta dirigida a la alta directiva 0 al representante del hotel y entrevistas aplicadas a los usuarios del servicio hotelero.

\section{Instrumentos}

La presente investigación se define como descriptiva con una estructura metodológica de enfoque mixto. Se generaron resultados directamente de la fuente de interés, a partir de encuestas aplicadas a los administradores hoteleros $y$ entrevistas semiestructuradas realizadas a los usuarios o clientes que consumen estos servicios.

La investigación se realizó en la ciudad de Portoviejo, Manabí, Ecuador en los meses de octubre y noviembre del 2019. Cabe indicar que los cuestionarios han sido diseñados con un espacio que conlleva los objetivos de la investigación, así mismo, se otorga 
un espacio para que el participante indique información relacionado al sexo, edad. En cuanto al diseño de los instrumentos, la encuesta cuenta con 11 preguntas, y la entrevista con 5 preguntas.

La estructura metodológica se aplicó de forma periódica durante la jornada laboral diurna de hoteles de la ciudad de Portoviejo, Manabí, Ecuador. Por su parte, los cuestionarios permitieron diagnosticar los inconvenientes a los que se enfrentan las administraciones hoteleras, en términos económicos, operativos y de servicios; además, se obtuvo información referente a las opciones de manejo que cuenta actualmente el sector turístico, específicamente en el manejo de los hoteles; esto en aras de proponer un modelo de gestión acorde a las necesidades de los clientes, pero que considere también la sostenibilidad y rendimiento empresarial. Se espera de esta forma que los establecimientos que adopten estas medidas tengan un efecto positivo que permita posicionar en el medio, establecimientos más competitivos y con mayor calidad en el servicio ofertado por la industria hotelera de la ciudad de Portoviejo, Manabí, Ecuador.

\section{Muestras}

Para la encuesta aplicada a los administradores y alta directiva de los establecimientos hotelero, la población total considerada en el estudio es 81 establecimientos, según el reporte de la Dirección de Turismo del GAD Portoviejo, Dirección Provincial de Turismo, propuesto en el año 2017.

Por su parte, las entrevistas fueron aplicadas en aras de receptar el número máximo de participantes, los cuales fueron seleccionados al azar de entre las personas que visitaban el establecimiento hotelero.

El tamaño de la muestra para las encuestas se determinó mediante la ecuación 1 (Aguilar-Barojas, 2005), considerando un intervalo de confianza del 95\% (representa un valor de $z=1,96)$ y un margen de error porcentual del $5 \%$.

$$
\boldsymbol{n}=\frac{N * z^{2} * p * q}{e^{2}(N-1)+z^{2} * p * q}
$$

Donde:

$\mathrm{n}$ : tamaño de la muestra.

$\mathrm{N}$ : tamaño de la población (81 establecimientos). 
z: valor de z crítico obtenido en función del nivel de confianza. En un $95 \%$ de confianza, el valor de $z$ es 1,96 .

p: proporción aproximada de la población que presenta la variable estudiada $(0,5)$.

q: proporción de la población que no presenta la variable estudiada $(p+q=1)$.

e: error muestreal (5\%).

$$
\begin{gathered}
\boldsymbol{n}=\frac{81 * 1,96^{2} * 0,5 * 0,5}{0,05^{2}(81-1)+1,96^{2} * 0,5 * 0,5} \\
n=67,04 \approx 67
\end{gathered}
$$

\section{Descripción de los participantes}

La muestra de participantes fue escogida independientemente del sexo y edad, de forma aleatoria en los establecimientos hoteleros de la ciudad de Portoviejo, Manabí, Ecuador. Cabe resaltar que, en algunos casos por la disponibilidad de tiempo de la alta directiva, no se los pudo encuestar, por consiguiente, fueron encuestados los representantes disponibles del hotel.

De las 67 personas encuestadas, 44 son de sexo masculino y 23 de sexo femenino, es decir, el $66 \%$ fueron varones y el restante $34 \%$ corresponde a mujeres. Los varones encuestados tienen una edad que oscila entre 36 años y 69 años; por su parte, las mujeres que fueron parte del estudio tienen una edad entre 28 y 51 años.

Respecto a las entrevistas, se aplicaron a 3 turistas hospedados en cada uno de los establecimientos visitados con fines investigativos. Del total de participantes (201 personas), el $63,68 \%$ de los participantes es de sexo femenino, mientras que el $36,32 \%$ restante de sexo masculino.

La discusión y revisión de la bibliografía permitió contrastar los resultados obtenidos a través de la aplicación de los instrumentos y reportes de instituciones públicas y privadas y de investigaciones de autores independientes.

\subsubsection{Evaluación de}

\section{potencialidades}

Se realizaron visitas de campo para analizar las potencialidades del sector hotelero en función de la información que ha generado el departamento de turismo del GAD Portoviejo. Mediante investigación bibliográfica se tomaron metodologías basadas en la evaluación del carácter y atributos 
del destino propuestas por Zambrano (2019).

\subsubsection{Presentación de resultados}

Esta fase consistió en diseñar estrategias para posicionar la gestión hotelera del medio, de modo que esto favorezca el posicionamiento de Portoviejo como destino turístico. A partir del análisis previo, se plantearon estrategias relacionadas con el desarrollo de actividades turísticas innovadoras, generación de ideas creativas y estrategias basadas en marketing, mediante una planificación para desarrollar una marca hotelera con su adecuada comercialización y posicionamiento en la provincia, en el país y para visitantes y turistas extranjeros.

\subsection{Diseño del modelo de gestión hotelera}

El modelo fue propuesto a partir de metodologías referentes que han sido planteadas en otras investigaciones. A partir de los procedimientos consultados, se discutió y propuso un modelo acorde a las necesidades y características del sector hotelero de Portoviejo. Las metodologías referenciadas fueron:
Castro et al., 2006; Cazañas et al., 2011; Cedeño, 2018.

\section{Resultados y discusión}

\subsection{Modelo de Gestión Hotelera}

El modelo de gestión aplicado a los establecimientos hoteleros de la ciudad de Portoviejo tiene como finalidad potenciar la actividad de este sector, optimizar recursos y aumentar la productividad de los hoteles de la ciudad. Cabe indicar que el modelo propuesto en este manuscrito surge a partir de la revisión de modelos planteados en investigaciones desarrolladas con fines similares a los del presente trabajo investigativo. De modo que, Cazañas et al., 2011 plantea un modelo estructurado con los requisitos propuestos para la implementación del Sistema de Gestión de la Calidad según la norma ISO 9001: 2015. Castro et al., 2006 presenta un modelo con componentes básicos como el diagnóstico inicial, definición del negocio, estrategias, organización, operatividad del modelo, control operacional y evaluación. Por su parte, Cedeño (2018) estructura un modelo que se basa principalmente 
en el análisis situacional, evaluación de los procesos aplicados por la industria hotelera y la definición de la documentación que guarda relación con la implementación del modelo de gestión.

El modelo de gestión hotelera para la ciudad de Portoviejo está compuesto por 3 etapas definidas de la siguiente forma: la primera fase consiste en la caracterización del estado actual del sector hotelero, con la respectiva evaluación de la gestión hotelera de los establecimientos ubicados en Portoviejo, Manabí, Ecuador. La segunda fase se basa en la estructura documental, descripción del modelo, identificación de procesos y estructura funcional. Por su parte, la tercera fase se fundamenta en la evaluación y mejora continua del sistema de gestión hotelera, mediante revisiones periódicas y auditorías internas.

\subsection{Fase 1 del modelo de gestión}

\subsubsection{Diagnóstico situacional turístico}

La ciudad de Portoviejo es la cabecera cantonal del cantón Portoviejo y capital de la provincia de Manabí. Esta ciudad se caracteriza por su movimiento comercial, industrial, cultural y gastronómico, puesto que recientemente fue reconocida como parte de la Red de Ciudades Creativas, en la categoría Gastronomía, por la Organización de las Naciones Unidas para la Educación, la Ciencia y la Cultura (UNESCO) (Ministerio de Turismo, 2019). Portoviejo cuenta con una población de 238430 habitantes según el Censo del INEC (2010) y entre sus características se resalta que se encuentra a 136 m.s.n.m. (metros sobre el nivel del mar), la temperatura promedio durante todo el año es $24^{\circ} \mathrm{C}$. La figura 1 muestra su ubicación geográfica.

En cuanto a la demanda turística, se reporta que el $41,5 \%$ de los participantes en la investigación manifiestan que llegan por descanso y vacaciones, mientras que el 25,5\% por negocios y trabajo, según la figura 2. Por su parte, Laaz (2018) reportó que durante los meses de julio-diciembre del 2018 , el $35,7 \%$ de los visitantes llegan hasta Portoviejo por razones de descanso. Esto permite concluir que las razones de los turistas para visitar Portoviejo son: en primer lugar, por descanso, seguido de actividades laborales o 
negocios y finalmente por conocer la

ciudad.

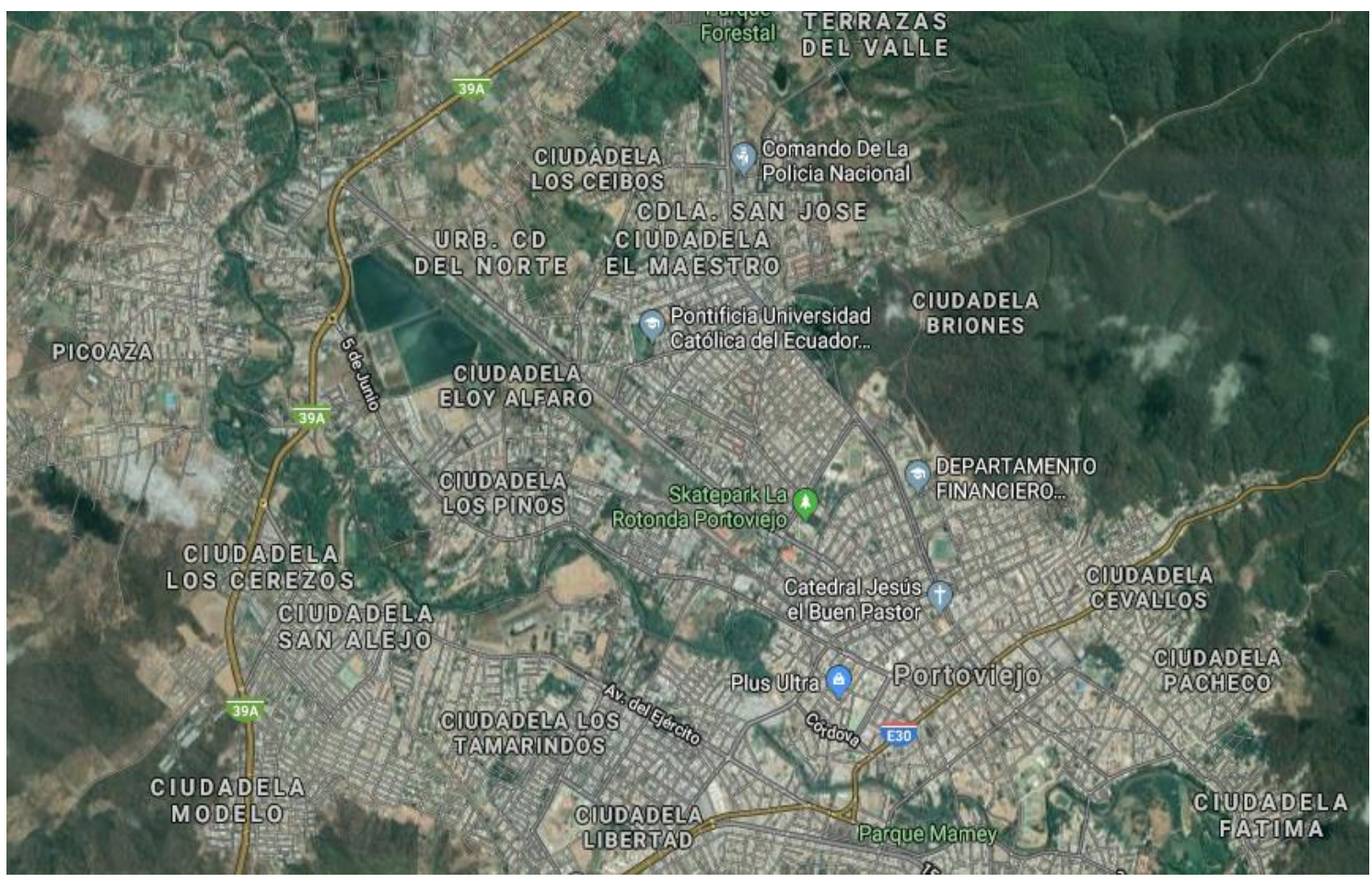

Figura 1. Ubicación geográfica del cantón Portoviejo

Según datos del GAD Portoviejo (2019), aproximadamente, se reciben 675 visitantes por día, que acuden entre otras cosas a realizar trámites, visitas turísticas y por motivos de trabajo. En este sentido, se ha determinado que, para los administradores de hoteles de la ciudad de Portoviejo, el cliente es el elemento más importante en la cadena productiva de la industria hotelera, denotándose que el 75;4\% concluye este resultado. El instrumento cualitativo permitió determinar que los clientes consideran que la calidad del servicio es buena, no obstante, todos los participantes consideran que hay puntos de mejora, especialmente en la atención al cliente, mayor difusión en medios digitales y la planificación desde la administración de los establecimientos hoteleros. 


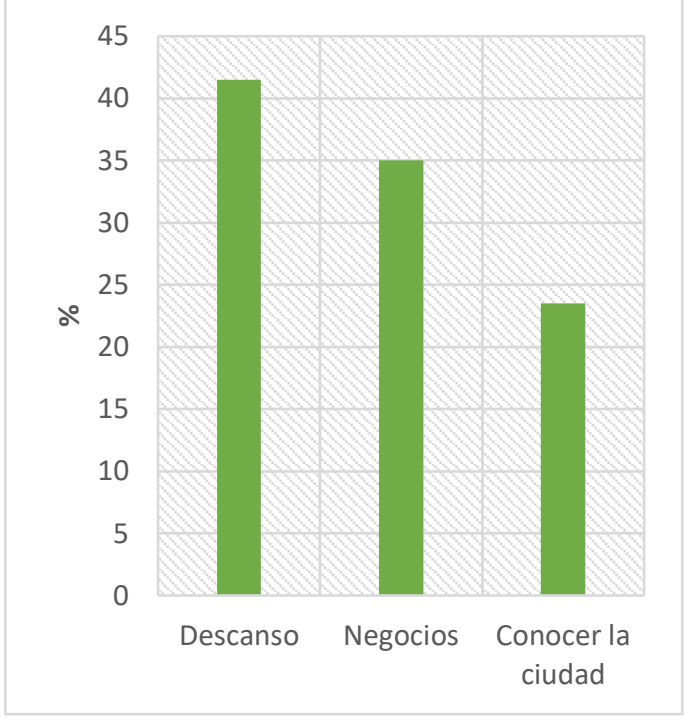

Figura 2. Razones de los turistas para visitar Portoviejo

Según la Dirección Municipal de Turismo del GAD Portoviejo en reporte realizado en 2017, esta ciudad cuenta con 198 establecimientos de alimentación. En cuanto al alojamiento, se han cuantificado 81 establecimientos en la ciudad de Portoviejo, de los cuales el $14,81 \%$ corresponde a hoteles, el $46,91 \%$ a hostales y el $19,61 \%$ a moteles. Cabe indicar que después del terremoto la planta hotelera de la ciudad disminuyó, debido al colapso de estructuras (Alcívar, 2018). Por otra parte, cabe señalar que una de las principales características de la ciudad es la resiliencia y por lo tanto este comportamiento se refleja en el sector turístico-hotelero.

\subsubsection{Análisis de la gestión hotelera}

Mediante el instrumento cuantitativo se determinó que el $66,35 \%$ de los participantes considera que la infraestructura hotelera de la ciudad es calificada como buena, el $23,2 \%$ como excelente y el restante $(10,45 \%)$ como regular. Por su parte, Alcívar (2018) reportó que Portoviejo cuenta actualmente con infraestructura turística que ha ido evolucionando y optimizándose en función del crecimiento de la ciudad. Se concluye que, si bien la gestión hotelera ha permitido potenciar la actividad turística de Portoviejo, aún no es suficiente para ofertar un servicio que cumpla todos los estándares de calidad.

Además, aspectos como la regeneración urbana de la ciudad constituyen ventajas sustanciales para la industria hotelera (Baum, 1990; Murphy et al., 1996), sin embargo, se requiere mayor aporte gubernamental en temas de gestión turística para que los establecimientos hoteleros de Portoviejo sean más atractivos para los turistas. Por su parte, los usuarios que aportaron en la investigación, indicaron que factores exógenos de la ciudad, como la contaminación ambiental y acústica, 
así como la mala distribución del parque automotor, los cuales son competencias gubernamentales, deben ser mitigados, puesto que afectan la calidad del servicio turístico y hotelero ofertado en la ciudad de Portoviejo. Asimismo, señalan que debe realizar un mayor número de eventos culturales y artísticos de primer nivel para atraer a la comunidad turística nacional e internacional.

Es de carácter imperioso que los gobiernos autónomos descentralizados cantonales como provinciales aporten herramientas en materia de formación y capacitación hacia la alta directiva y los colaboradores y trabajadores de los hoteles, puesto que, gran parte de los participantes de la presente investigación señalan que no han recibido formación en estas temáticas; el $63,89 \%$ de los encuestados concuerda con el resultado señalado.

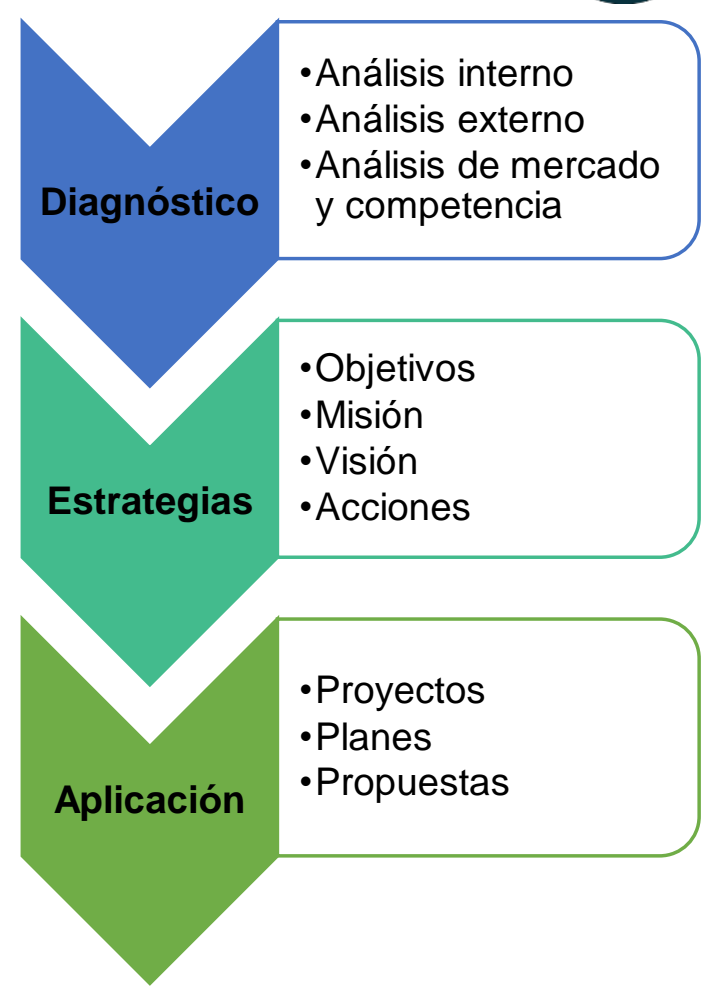

Figura 3. Etapas de la planificación y gestión hotelera

Tanto la planificación como la gestión hotelera y turística guardan relación con parámetros que en muchos casos son subestimados y por lo tanto no se ha alcanzan los niveles deseados en la operatividad, rendimiento y sostenibilidad de los hoteles (Waligo et al., 2013). Damián \& Suárez-Barraza (2015) reportan aspectos clave que deben considerarse en la estructura de una propuesta que permita potenciar la gestión hotelera de la ciudad de Portoviejo.

Se mencionan las siguientes estrategias para potenciar el sector 
turístico y la gestión hotelera de la ciudad de Portoviejo:

- Garantizar un abastecimiento permanente de servicios básicos como energía eléctrica, agua potable, red de alcantarillado, sistemas de vigilancia policial, tratamiento y gestión de residuos domésticos e industriales, acceso a internet, infraestructura para personas con capacidades especiales, vías de acceso y señalización.

- Infraestructura y zonas verdes que diversifiquen.

- Creación de centros de información turística en diversos puntos de la ciudad de Portoviejo.
- Proyectos de capacitación y formación en temáticas de administración y gestión hotelera y procesos de gestión de la calidad basados en la mejora continua.

- Generación de espacios culturales, gastronómicos, tecnológicos que sean atractivos para la comunidad turística.

- Potenciar el uso de herramientas tecnológicas que permitan establecer una red de negocios hoteleros y que favorezcan la accesibilidad y posicionamiento de los establecimientos.

\subsubsection{Matriz FODA}

Tabla 1.

Matriz FODA

\begin{tabular}{|c|c|}
\hline Fortalezas & Oportunidades \\
\hline $\begin{array}{l}\text { - } \text { Alta directiva y personal implicado con } \\
\text { el sistema de gestión hotelera. } \\
\text { - } \text { Plantilla de trabajadores en constante } \\
\text { capacitación y formación turística. } \\
\text { - Empoderamiento de los hoteles de la } \\
\text { ciudad. } \\
\text { - Trayectoria de los establecimientos } \\
\text { - hoteleros de la ciudad. } \\
\text { Establecimientos hoteleros con } \\
\text { nuevos servicios y productos } \\
\text { disponibles en el mercado. } \\
\text { - Uso de nuevas tecnologías } \\
\text { informáticas. } \\
\text { Instalaciones que cumplen con los } \\
\text { requisitos mínimos para garantizar un } \\
\text { servicio de calidad. }\end{array}$ & $\begin{array}{l}\text { - Excelente ubicación geográfica para } \\
\text { la actividad hotelera. } \\
\text { - La ciudad cuenta con recursos } \\
\text { gastronómicos, históricos y culturales } \\
\text { que son atractivos para los turistas. } \\
\text { - Potencial mercado de clientes. } \\
\text { - Aumento de las actividades turísticas } \\
\text { en el Ecuador con nuevos servicios y } \\
\text { destinos. }\end{array}$ \\
\hline
\end{tabular}




\section{Debilidades}

Amenazas

- Poco conocimiento en el área de salud e higiene por parte de los colaboradores.

- Escasa implementación de procedimientos que fomenten la gestión hotelera en la ciudad.

- Ausencia de metodologías de evaluación y auditoría interna.

- Resistencia a procesos de cambios.

- Aspectos administrativos.

- Falta de experticia respecto a sistema de gestión de seguridad y salud.

\subsection{Fase 2 del modelo de gestión hotelera}

\subsubsection{Introducción}

Los hoteles de la ciudad de Portoviejo presentan inconvenientes en cuanto a la gestión administrativa y estratégica en base a la calidad del servicio y la restauración de la infraestructura con la que se cuenta. Por tanto, es imperiosa la necesidad de aplicar procesos de gestión para complementar los servicios y productos ofertados por los hoteles de la ciudad. Por otra parte, esto permitirá mejorar la rentabilidad de los proyectos hoteleros del sector y desarrollar la actividad turística.
- Escasez de formación en áreas específicas de la gestión hotelera en la ciudad de Portoviejo.

- Disminución de la demanda.

- Aumento de la oferta en forma de competidores informales que ofertan servicios de alojamiento.

- Contaminación ambiental, exceso de ruido, parque automotor y trafico concentrado en la ciudad.

- La mayoría de los hoteles cuentan con una estacionalidad definida.

- Los precios de los servicios ofertados por el sector hotelero de la ciudad son inestables.
El objetivo del presente modelo de gestión es lograr la debida articulación de todos los componentes que estructuran un modelo de gestión acorde a las necesidades del sector hotelero, garantizando el cumplimiento de los estándares de calidad y la mejora continua de los procesos administrativos y estratégicos.

\subsubsection{Definición del modelo}

El modelo cuenta en su primera etapa con la caracterización del sector turístico y hotelero de Portoviejo y consecuentemente con la valoración de la gestión aplicada en los establecimientos de alojamiento, mediante instrumentos de investigación que permitieron 
recolectar información directa y confiable. Esta información fue analizada mediante la estrategia FODA, que plantea las necesidades del sector, partes interesadas, procesos de gestión, además, se determinó las pautas que deben ponerse en práctica para la mejora del servicio de alojamiento. Las estrategias mencionadas deben aplicarse para potenciar la gestión a corto y mediano plazo.

\subsubsection{Generalidades}

\section{Misión}

Generar un servicio hotelero acorde a las necesidades de los turistas nacionales e internacionales que visitan la ciudad de Portoviejo, de modo que los establecimientos hoteleros aseguren la rentabilidad y competitividad en el mercado turístico y puedan aplicar estrategias de gestión administrativa, estratégica en base a requisitos de calidad y restauración de la infraestructura física.

\section{Visión}

Consolidar a los establecimientos hoteleros de Portoviejo como un sector que desarrolle procesos de gestión basados en la evaluación y mejora continua de procedimiento que permitan potenciar el turismo a nivel nacional, regional e internacional con lineamientos estandarizados que resalten el prestigio del servicio ofertado y el cumplimiento de los requerimientos de los usuarios.

\subsubsection{Estructura organizacional y funciones}

La estructura organizacional para un establecimiento hotelero basado en el modelo de gestión propuesto, se basa en los requisitos propuestos en las normas INEN (2008) para el sector de Alojamiento.

\section{Tabla 2.}

Ocupaciones, funciones y competencias laborales del sector alojamiento en base a las normas INEN

\begin{tabular}{|l||l|}
\hline \multicolumn{1}{|c|}{ Ocupación } & $\begin{array}{c}\text { Código Norma } \\
\text { INEN }\end{array}$ \\
\hline $\begin{array}{l}\text { Encargado de } \\
\text { reservas }\end{array}$ & $\frac{\text { NTE INEN 2428: }}{2008}$ \\
\hline \hline Recepcionista & $\frac{\text { NTE INEN 2429: }}{\underline{2008}}$ \\
\hline \hline Ama de llaves & $\frac{\text { NTE INEN 2430: }}{\underline{2008}}$ \\
\hline \hline Botones & $\frac{\text { NTE INEN 2431: }}{2008}$ \\
\hline \hline Camarera de pisos & $\frac{\text { NTE INEN 2432: }}{2008}$ \\
\hline
\end{tabular}




\begin{tabular}{|c|c|}
\hline $\begin{array}{l}\text { Servicio } \\
\text { personalizado de } \\
\text { información turística }\end{array}$ & $\frac{\text { NTE INEN 2433: }}{\underline{2008}}$ \\
\hline $\begin{array}{l}\text { Encargado de } \\
\text { mantenimiento }\end{array}$ & $\frac{\text { NTE INEN 2434: }}{\underline{2008}}$ \\
\hline $\begin{array}{l}\text { Recepcionista } \\
\text { polivalente }\end{array}$ & $\frac{\text { NTE INEN 2446: }}{\underline{2008}}$ \\
\hline Jefe de recepción & $\frac{\text { NTE INEN 2447: }}{\underline{2008}}$ \\
\hline $\begin{array}{l}\text { Coordinador de } \\
\text { eventos }\end{array}$ & $\frac{\text { NTE INEN 2448: }}{\underline{2008}}$ \\
\hline Auditor nocturno & $\frac{\text { NTE INEN 2449: }}{\underline{2008}}$ \\
\hline $\begin{array}{l}\text { Administrador de } \\
\text { empresas de } \\
\text { alojamiento }\end{array}$ & $\frac{\text { NTE INEN 2450: }}{\underline{2008}}$ \\
\hline
\end{tabular}

Fuente: INEN, 2008.

\subsection{Fase 3 del modelo de gestión}

La evaluación y mejora continua constituye una fase obligatoria en todo sistema de gestión, puesto que de esta forma se determinan las fallas y se plantean acciones para corregir el proceso de gestión, por tanto, el modelo de gestión tiene una secuencia cíclica, en la cual constante y periódicamente se realiza la mejora continua planteada en el ciclo de Deming (Castellani \& Sala, 2010). Para evaluar el cumplimiento de los objetivos propuestos se plantean indicadores basados en la Herramienta del Sistema Europeo de Indicadores Turísticos Sostenibles, ETIS
(Comisión Europea, 2016). En este sentido, los indicadores propuestos son:

- Número (porcentaje) de hoteles que emplean modelos de gestión hotelera: 0 - 100\%.

- Número (porcentaje) de establecimientos hoteleros que generan mecanismos para evaluar y darle seguimiento a los procesos de gestión: 0 - 100\%.

- Número (porcentaje) de establecimientos hoteleros que han sido certificados por sus modelos de gestión: 0 - 100\%.

- Número (porcentaje) de establecimientos hoteleros que generan mecanismos para evaluar y darle seguimiento a los procesos de gestión: 0 - 100\%.

- Número (porcentaje) de auditorías internas aplicadas en los establecimientos hoteleros que emplean modelos de gestión: $0-100 \%$.

- Número (porcentaje) de usuarios que registran más de una visita en el mismo establecimiento hotelero: 0 - 100\%.

- Número (porcentaje) de usuarios que resultan satisfechos con el servicio ofertado por el hotel: 0 $100 \%$. 
- Número (porcentaje) de establecimientos hoteleros que capacitan periódicamente a sus colaboradores: $0-100 \%$.

- Número (porcentaje) de establecimientos hoteleros que realizan tratamiento integral de residuos: $0-100 \%$.

- Número (porcentaje) de reportes favorables en base a estadísticas de organismos oficiales adscritos al MINTUR: 0 - 100\%.

\section{Conclusiones}

En la presente investigación se propone un modelo de gestión hotelera para la ciudad de Portoviejo. Esta propuesta fomenta una cultura de planificación, gestión administrativa y estratégica que considera políticas de mejora continua en base a metas definidas para un determinado período de tiempo. El modelo en mención ha sido construido en base a las necesidades y expectativas del sector hotelero, así como las características del medio; por lo tanto, permite mejorar la organización estructural a la que deben adaptarse los organigramas de los establecimientos hoteleros, así como la misión y visión del modelo de gestión, en aras de garantizar la sostenibilidad turística. Los requisitos mínimos exigidos para la implantación del modelo de gestión hotelera se fundamentan en los lineamientos propuestos en las normas INEN, con la finalidad de estandarizar los procesos, competencias y funciones de las partes involucradas en el modelo.

A nivel gubernamental, Ecuador ha tomado medidas para potenciar las actividades turísticas, no obstante, aún existen falencias en cuanto a la organización, planificación y gestión hotelera. El presente estudio busca aportar estrategias que con su debida aplicación mejoren la calidad de los servicios y a su vez optimicen la rentabilidad y sostenibilidad de los establecimientos hoteleros. En este sentido, la ciudad de Portoviejo cuenta con una ubicación y características favorables, sin embargo, el servicio brindado debe potenciarse tanto desde los entes gubernamentales e internamente desde la alta directiva de los hoteles aplicando procesos de mejora continua en términos de operación, servicios y productos ofertados a los clientes.

La planta hotelera de Portoviejo debe fortalecerse en aspectos como 
infraestructura y capacitación. En este sentido, las instituciones gubernamentales como el Ministerio de Turismo, Gobierno Provincial de Manabí y GAD Portoviejo deben promover capacitaciones en temas de gestión y administración y paralelamente, generar condiciones exógenas como seguridad, servicios básicos, que conviertan a la industria hotelera de la localidad en un sector atractivo para los usuarios y turistas.

\section{Bibliografía}

Agatón, D. Cruz, A. (2015). Identificación y evaluación del espacio turístico potencial de la zona poniente de Acapulco y su repercusión en el desarrollo local. México. P. 5-8. Recuperado de http://ru.iiec.unam.mx/3014/ 1/Eje11-123-AgatonCruz.pdf

Aguayo-Moral, J. M. (1997). Un modelo para la segmentación de la información contable en las empresas hoteleras. Tesis doctoral. Universidad de las Palmas de Gran Canaria
Aguilar-Barojas, S. (2005). Fórmulas para el cálculo de la muestra en investigaciones de salud. Salud en Tabasco, 11(1-2), 333-338.

Albuja, A. (2016). Evaluación del potencial turístico del cantón San Miguel de Urcuquí para dinamizar la actividad turística del sector. Trabajo de grado previo a la obtención del título de Ingeniería en Gestión Turística y Hotelera. Chimborazo. Ecuador. pp. 32-33. Recuperado de http://dspace.unach.edu.ec/ bitstream/51000/803/1/UNA CH-EC-IG.TUR20160005.pdf

Alcívar, G. (2018). Zonificación funcional turística para la reactivación post terremoto del turismo en la Ciudad de Portoviejo, Manabí. Tesis previa la obtención del título de Ingeniera en Turismo. ESPAM MFL. Calceta, Ecuador. Recuperado de: http://repositorio.espam.edu .ec/bitstream/42000/943/6/T T88.pdf 
Batista, E., González, J., González, I., Leyva, E. (2017). Procedimiento para medir la imagen de destinos turísticos. Ciencias Holguín, 23(3). 1-18. Recuperado de http://www.redalyc.org/articu lo.oa?id=181552082001

Baum, T. (1990). Competencies for hotel management: Industry expectations of education. International Journal of Contemporary Hospitality Management, 2(4).

Castellani, V., \& Sala, S. (2010). Sustainable performance index for tourism policy development. Tourism management, 31(6), 871880.

Doi: https://doi.org/10.1016/j.tour man.2009.10.001

Castelló, E. (1993). Nuevas tendencias en contabilidad de gestión: Implantación en la empresa española. A.E.C.A.: Madrid. pp.135165.

Castro, M., Flamenco, M., \& Rivas, J. (2006). Diseño de un modelo de gestión de destinos turísticos sostenibles para mejorar la afluencia de visitantes de los pequeños hoteles de la Zona Oriental del Salvador. Tesis de pregrado. Licenciatura en Administración de Empresas. UFG. EI Salvador-San Salvador. p. 165. Recuperado de: http://ri.ufg.edu.sv/jspui/bitst ream/11592/6714/5/338.479 1C353dCapitulo\%20IV.pdf

Cazañas-Rivero, M., GonzálezGonzález, A., \& de los Ángeles González-Solá, M. (2011). Diseño de un Sistema de Gestión de la Calidad en el proceso de alojamiento en el Hotel "Gran Caribe Villa Tortuga". Ingeniería Industrial, 32(1), 37-42. Recuperado de: https://www.redalyc.org/pdf/ 3604/360433575006.pdf

Cedeño, J. (2018). Modelo de gestión hotelera para la mejora del servicio de alojamiento en la ciudad de Manta. Tesis previa la obtención del título de Ingeniera en Turismo. ESPAM MFL. Calceta, Ecuador. Recuperado de: 
http://repositorio.espam.edu

.ec/bitstream/42000/734/1/T

T83.pdf

Comisión Europea. (2016). El

Sistema Europeo de Indicadores Turísticos.

Herramienta del ETIS para la gestión de destinos sostenibles. Luxemburgo: Unión Europea.

Damian, I. E., \& Suárez-Barraza, M. F. (2015). Innovación de procesos en la gestión turística: Una revisión de la literatura. Intangible Capital, 11(1), 147-165.

GAD Portoviejo. (2016). Actualización del Plan de desarrollo y ordenamiento territorial del cantón Portoviejo 2015. Portoviejo, Ecuador.

GAD Portoviejo. (2017). Generación de Instrumentos de gestión para la sostenibilidad del Plan (POT, 2015). Portoviejo, Ecuador.

Huéscar, A. (1993): "Nuevo marco conceptual del turismo". Estudios turísticos. Instituto de Estudios Turísticos D.G. de Política Turística, no117 pp. 23-48.

INEC. (2010). Base de DatosCenso de Población y Vivienda 2010. Instituto Nacional de Estadística y Censo. Quito, Ecuador. Recuperado de: https://www.ecuadorencifras .gob.ec/base-de-datoscenso-de-poblacion-yvivienda-2010/

INEN. (2008). Turismo, Competencia Laboral, Alojamiento. Quito, Ecuador: INEN. Recuperado de: http://apps.normalizacion.go b.ec/descargas/vista/public/

Laaz, G. (2018). Plan de desarrollo turístico post terremoto para la mejora del turismo en el cantón Portoviejo. (Tesis para la obtención del título de Ingeniero en Turismo). ESPAM MFL, Calceta, Ecuador.

Ministerio de Turismo. (2018). Boletín enero 2018. Recuperado de http://servicios.turismo.gob. ec/descargas/Turismocifras/ 
Boletin/Boletin_enero-

2018.pdf

Ministerio de Turismo. (2019).

Portoviejo un destino gastronómico reconocido en el mundo. Recuperado de https://www.turismo.gob.ec/ portoviejo-un-destinogastronomico-reconocidoen-el-mundo/

Murphy, J., Forrest, E. J., Wotring, C. E., \& Brymer, R. A. (1996). Hotel management and marketing on the Internet: An analysis of sites and features. The Cornell Hotel and Restaurant Administration Quarterly, 37(3), 7-82.

Musa, A., \& Sanchís, P. (1993). Determining accommodation prices in the hotel industry. Estudios Turísticos, 117, 4960.

ONU. (1994): Recomendaciones sobre estadísticas del turismo, número de venta S.94.XVII.6., ed. Naciones Unidas, Nueva York, p.5. Departamento De Información Económica y Social y Análisis de
Políticas. División de Estadística, Organización de las Naciones Unidas.

Ruhanen, L. (2008). Progressing the sustainability debate: A knowledge management approach to sustainable tourism planning. Current issues in tourism, 11(5), 429455.

Waligo, V. M., Clarke, J., \& Hawkins, R. (2013). Implementing sustainable tourism: A multi-stakeholder involvement management framework.

Tourism management, 36, 342-353.

Zambrano, A. (2019). Evaluación de las potencialidades de la ciudad de Manta para su definición como destino turístico. Trabajo de grado previo a la obtención del título de Ingeniería en Turismo. ESPAM MFL, Calceta, Ecuador. pp. 42. 
Recuperado de

http://repositorio.espam.edu

.ec/bitstream/42000/1023/1/

TTT10.pdf 